\title{
ACTIVATED MULTI-WALLED CARBON NANOTUBES FOR ELECTROCHEMICAL DETECTION OF DOPAMINE IN THE PRESENCE OF ASCORBIC AND URIC ACID
}

\author{
ANDREA KELLENBERGER ${ }^{a}$, RODICA GAVRILA ${ }^{a}$, \\ NICOLETA PLESU $\mathbf{b}^{*}$
}

\begin{abstract}
Electrochemical sensors have been prepared using pristine and activated multi-walled carbon nanotubes on glassy carbon electrode. The detection of dopamine in the presence of ascorbic acid was tested by square wave voltammetry and cyclic voltammetry. Best results were obtained for glassy carbon modified with activated carbon nanotubes electrodes. A linear dependence between current intensities and dopamine concentrations is found in both the absence/presence of ascorbic acid, in the range of 4-100 $\mu \mathrm{M}$ / 6-100 $\mu \mathrm{M}$, with detection and quantification limits of $0.44 / 0.64$ and 1.45 / $2.14 \mu \mathrm{M}$, respectively and high sensitivity. The developed electrodes also showed very good performance in separating the oxidation potentials of ascorbic acid, dopamine and uric acid, with peak potential differences of 200 and $170 \mathrm{mV}$. Dopamine detection in synthetic solutions in the presence of both ascorbic and uric acid gave recovery rates of $98 \%$, indicating that the method is reliable.
\end{abstract}

Keywords: electrochemical sensor, carbon nanotubes, dopamine, ascorbic acid, square wave voltammetry

\section{INTRODUCTION}

Dopamine (DA) and ascorbic acid (AA) are organic compounds with biomedical importance. Neurotransmitters are chemicals essential in the transfer of information between the neuron cells. Dopamine is a neurotransmitter present in mammalian brain tissue. Low dopamine levels have been associated with disorders such as schizophrenia and Parkinson's disease or addictions [1].

a Politehnica University Timisoara, Faculty of Industrial Chemistry and Environmental Engineering, 6 Vasile Parvan Bd., RO-300223, Timisoara, Romania

b "Coriolan Dragulescu" Institute of Chemistry, Bd. Mihai Viteazul 24, RO-300223, Timisoara, Romania

*Corresponding author: plesu_nicole@yahoo.com 
The concentration of DA in the extracellular fluid of the brain of healthy people is in the submicromolar range, but it is lower for people suffering from the aforementioned disorders. Dopamine usually exists in cationic form at physiological $\mathrm{pH}$ and is oxidized to quinone form (DAQ) in a two-proton / twoelectron process. Ascorbic acid is an antioxidant with an important role in preventing infectious diseases. AA has been studied in the context of oxidative stress associated with cancer and other diseases. AA is also used in preserving food and beverages. At physiological $\mathrm{pH}, \mathrm{AA}$ exists as the monodeprotonated ascorbate anion and it is oxidized to dehydro-ascorbic acid in a process involving two electrons and a proton [2].

The concentration of DA in the cerebral extracellular fluid is in the submicromolar domain in the case of healthy people, but it can also reach lower values in the case of diseases such as Parkinson's disease. The AA concentration is in the range of $0.2-0.5 \mathrm{mM}$.

Two of the most widely used techniques for in vivo monitoring of neurotransmitters are microdialysis and fast-scan cyclic voltammetry (FSCV). In microdialysis, a semi-permeable probe is injected into the brain and the analyte that is present in the brain is perfused through the probe and collected for chemical analysis using techniques such as high-performance liquid chromatography (HPLC) with mass spectrometry (MS) or fluorescence as a detector [3]. The main disadvantage of microdialysis is the temporal low resolution. FSCV is a suitable technique for in vivo neurotransmitter detection, which presents a high temporal resolution [4].

DA and AA coexist in extracellular fluids and have similar oxidation potentials, especially on conventional electrodes, which results in an overlap of their voltammetric response. In this respect, electrode materials capable of oxidizing DA or AA in distinct potential windows would be most suitable for the selective detection of DA in the presence of AA. The main difficulty in electrochemical detection of DA is the presence of interfering molecules such as ascorbic acid and uric acid (UA). These molecules have an oxidation potential very close to that of DA. This issue can be controlled by modifying the electrode with suitable materials. Carbon-based materials (polymers) and noble metal nanoparticles (rhodium, palladium, iridium, platinum or gold) are suitable materials for electrode modification [5]. Polymer-based dopamine sensors based on conducting polymers such as poly 3,4-ethylenedioxythiophene (PEDOT) [6], polypyrrole (PPy) [7] poly-o-phenylenediamine (P-o-PD) [8], polyanilines (PANI) [9] show promising biocompatibility and stability. Regarding carbon nanomaterials, carbon nanotubes (CNT) and graphene are used in most cases in electrocatalysis due to their stable physical and chemical properties, large specific surface area, good conductivity, biocompatibility and synergistic effect on catalytic activity. Thus, vitreous carbon electrodes modified with $\mathrm{C}_{60}$ 
fullerenes were prepared followed by the constant potential deposition of platinum, which allowed the simultaneous detection of ascorbic acid, dopamine and uric acid, by separating their oxidation potentials [10]. The use of carbon paste electrodes modified with carbon nanotubes decorated with iron nanoparticles allowed the simultaneous detection of uric acid in the presence of interfering molecules such as ascorbic acid, dopamine and L-tyrosine [11]. Graphite-epoxy composites with synthetic zeolites have been successfully used for miniaturization of DA sensors [12]. Promising results have been obtained by modifying the electrodes with nanoparticles of noble metals, especially gold, due to its conductivity, catalytic activity and biocompatibility. For example, the electrochemical deposition of gold on the surface of reduced graphene oxide allowed the simultaneous, selective and sensitive detection of AA, DA and UA [13]. Also, the modification of carbon nanotubes with gold nanoparticles deposited by a chemical method had the effect of separating the oxidation potentials for AA, DA and UA [14]. Carbon nanotubes (multi-walled carbon nanotubes and single-walled carbon nanotubes) are nanomaterials with properties such as extraordinary tensile strength, excellent electrical conductivity and high chemical stability. The unique electronic properties of carbon nanotubes show better electron transfer rates when used as electrode materials and offer excellent prospects for the development of miniaturized electronic devices and electrochemical sensors [15]. Graphene is another carbon material, which presents excellent conductivity, stability, and low cost. The large surface area offered by the graphenes is useful for chemical detection [16]. Modified electrodes with reduced graphene oxide showed ultrasensitive detection of dopamine $[17,18]$.

The aim of the present study is to reveal the effect of multi-walled carbon nanotube (CNT) and activated multi-walled carbon nanotube (CNT/ACT) toward DA oxidation and to evaluate the electrochemical response in the presence of AA and UA. Even though CNT in various forms have been extensively utilized for DA sensors, our goal was to identify at what extent the activation process of CNT contributes to the enhancement of electrocatalytic activity towards DA oxidation.

\section{RESULTS AND DISCUSSION}

\section{Electrochemical detection of DA and AA on GC electrode}

Firstly, the electrochemical behavior of GC electrode was investigated in $0.1 \mathrm{~mol} \mathrm{~L}^{-1}$ PBS electrolyte support $(\mathrm{pH}=7.4)$. The square wave voltammograms (SWVs) recorded on GC for different dopamine concentrations 
(Figure 1a) show the appearance of an oxidation peak of DA at $0.33 \mathrm{~V}$ whose intensity increases with increasing DA concentration. In addition, its position shifts slightly from $0.33 \mathrm{~V}$ to $0.36 \mathrm{~V}$ with increasing DA concentration. SWVs give a linear calibration curve for dopamine (Figure 1b). The kinetics of DA oxidation reaction was studied by cyclic voltammetry for the highest value of DA concentration, by recording cyclic voltammograms (CVs) at different scan rates (Figure 1c). A well-defined oxidation peak is observed at potential values of $0.25 \mathrm{~V}$ that slightly shifts to more positive values $(0.27 \mathrm{~V})$ as the scan rate increases. The effect of scan rate on the current peak density is presented and discussed in Figure $4 \mathbf{d}$ and Table 1 for the oxidation of DA and $A A$ on $G C$ and $G C / C N T$ electrodes.
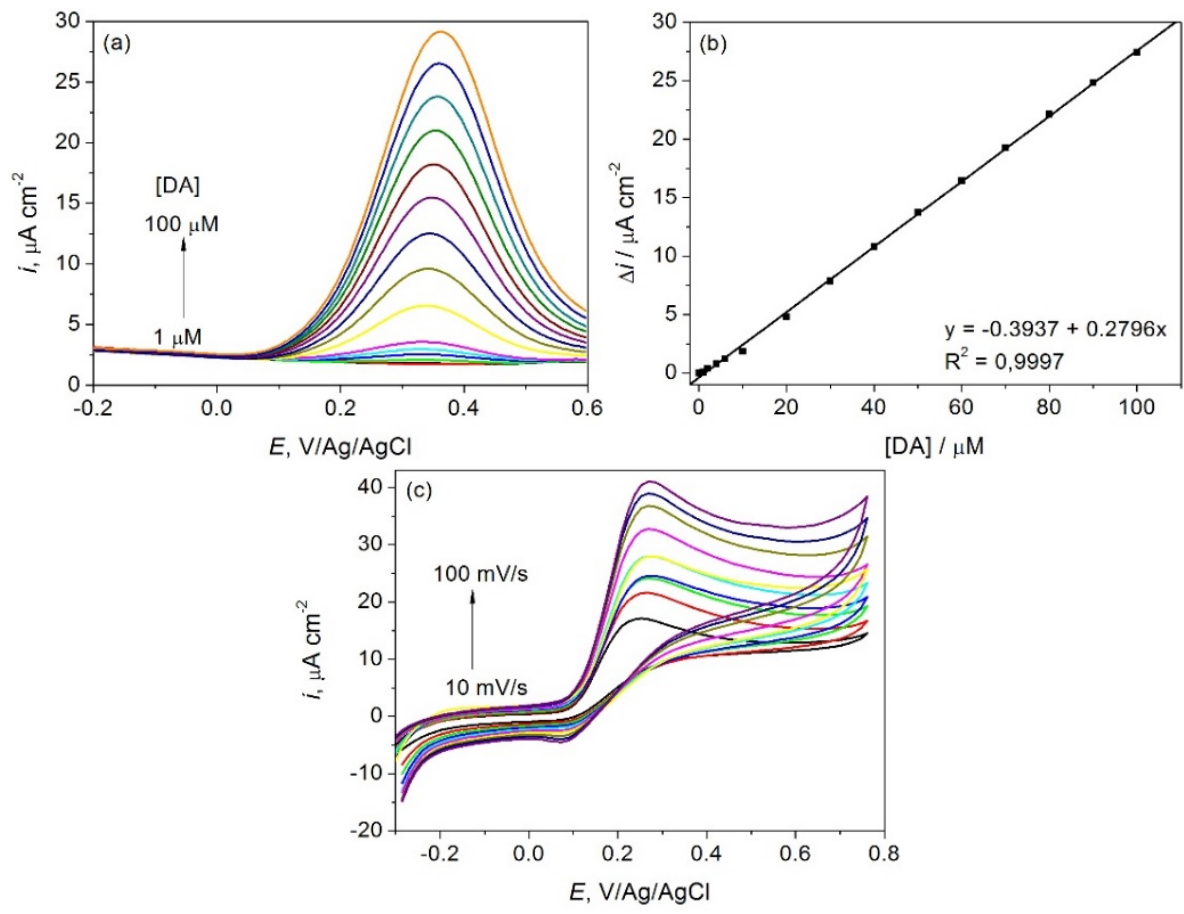

Figure 1. DA detection in $0.1 \mathrm{~mol} \mathrm{~L}^{-1} \mathrm{PBS}(\mathrm{pH}=7.4)$ on $\mathrm{GC}$ electrode:

(a) SWVs at different DA concentrations; (b) calibration curve and

(c) CVs in $100 \mu \mathrm{M}$ DA solution at different scan rates.

Square wave voltammograms recorded on GC for different concentrations of AA (Figure 2a) show that the intensity of the oxidation peak of AA is concentration dependent and its position shifts slightly from $0.40 \mathrm{~V}$ to $0.44 \mathrm{~V}$ with increasing concentration of AA. The obtained calibration line for AA on GC is 
illustrated in Figure $\mathbf{2 b}$. The kinetics of the AA oxidation reaction studied by cyclic voltammetry in $2 \mathrm{mM}$ AA solution, at different scan rates reveal an irreversible oxidation peak around $0.61 \mathrm{~V}$ (Figure $2 \mathrm{c}$ ). The position of this peak changes from $0.61 \mathrm{~V}$ to $0.68 \mathrm{~V}$ as the scan rate increases. Since the oxidation potentials of DA and AA differ by only $70 \mathrm{mV}$, their simultaneous detection on GC electrode is restricted, as proven by square wave and cyclic voltammograms given in Figure 3 for a mixture of AA and DA.
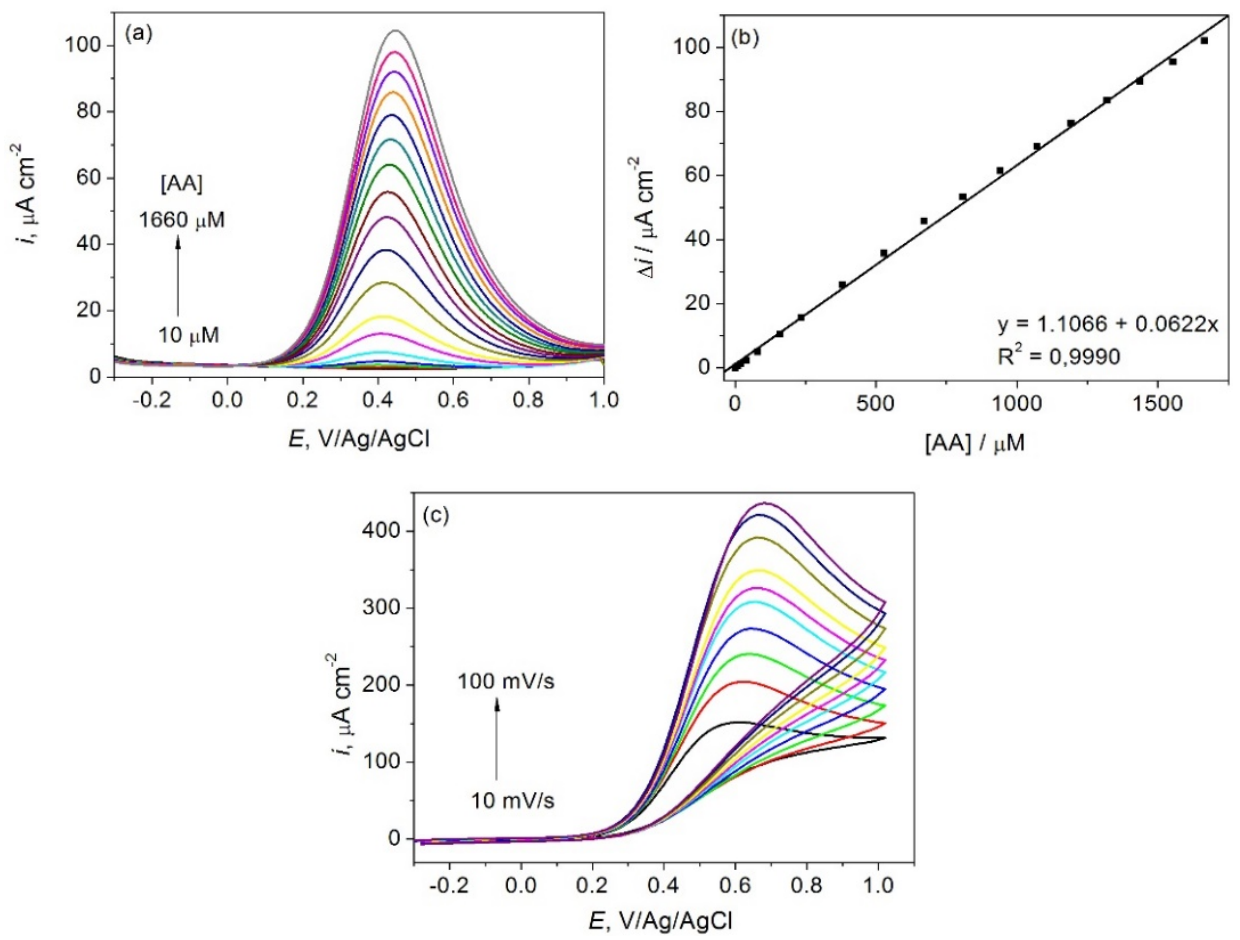

Figure 2. AA detection in $0.1 \mathrm{M}$ PBS $(\mathrm{pH}=7.4)$ on $\mathrm{GC}$ electrode: (a) SWVs at different AA concentrations; (b) calibration curve and (c) CVs in 2 mM AA solution at different scan rates.

It is observed that when DA is added to the AA solution in PBS, the peak current intensity increases for both cyclic and square wave voltammogram, but there is no clear differentiation of the two-oxidation processes, these being overlapped due to the closed potentials at which they occur. 

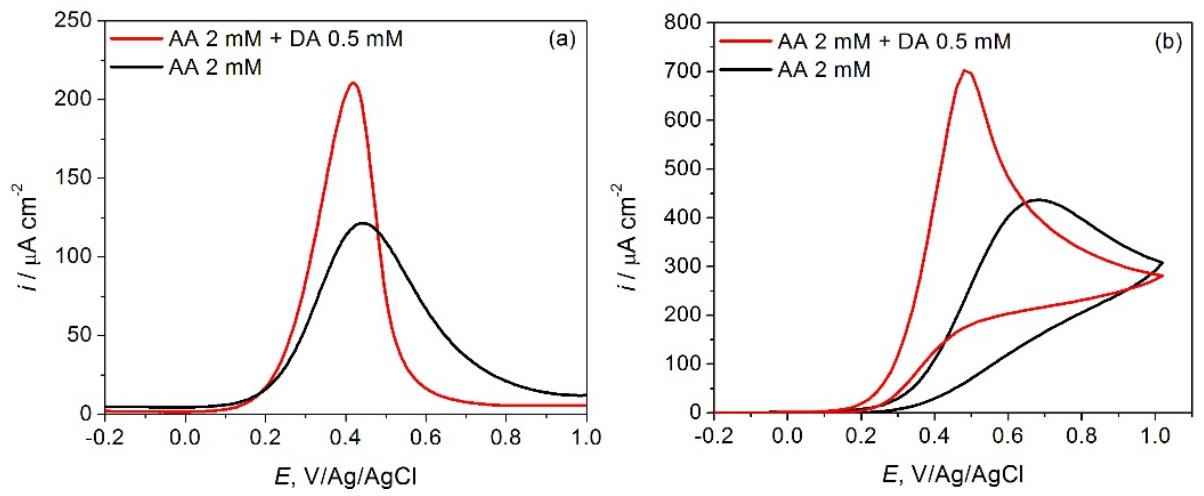

Figure 3. Simultaneous detection of AA and DA in 0.1 M PBS ( $\mathrm{pH}=7.4)$ on GC electrode: (a) square wave and (b) cyclic voltammograms.

\section{Electrochemical detection of DA and AA on GC/CNT electrode}

To improve the electrochemical response, the GC electrode was modified with carbon nanotubes. The SWVs recorded on GC/CNT for different DA concentrations (Figure 4a) reveal an oxidation peak situated at $0.13 \mathrm{~V}$, whose intensity increases linearly with increasing DA concentration (Figure 4b). The oxidation potential of DA on the GC/CNT electrode decreases compared to GC, which proves its increased activity for DA oxidation. The calibration line obtained is linear for concentrations between 1 - $100 \mu \mathrm{M}$. Cyclic voltammograms (Figure 4c) show a reversible oxidation peak at a potential value of $0.16 \mathrm{~V}$, slightly shifting to more positive values as the scan rate increases.

Information about the kinetics of DA and AA oxidation on GC and GC/CNT electrodes are obtained from the slope of the linear dependence of $\log i_{p}$ versus $\log v$, were $i_{p}$ is the peak current density from the CVs and $v$ is the scan rate (Figure $\mathbf{4 d}$ ). The oxidation of DA and AA on GC electrode is a fast electron transfer reaction, controlled only by diffusion, as indicated by the slope close to 0.5 , which is the theoretical value for a process controlled by diffusion (Table 1). On GC/CNT electrode, the linear dependence of log $i_{\mathrm{p}}$ versus $\log v$ for $\mathrm{AA}$ oxidation exhibits a slope close to 0.5 , indicating that the reaction is still controlled by diffusion, whereas for DA oxidation the slope changes to 0.93 , close to the theoretical value of 1 , proving a surface adsorption-controlled kinetics. 

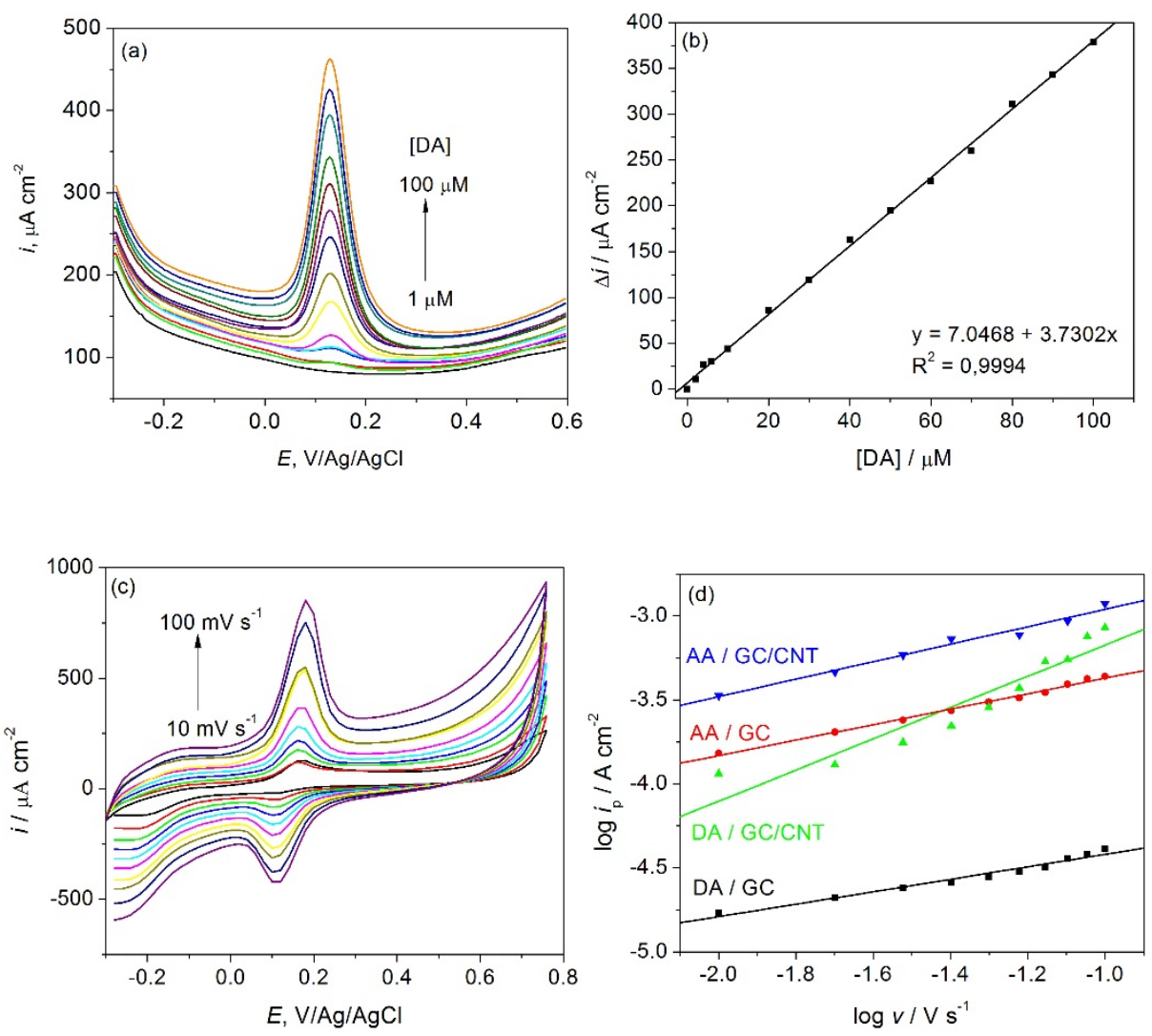

Figure 4. DA detection in $0.1 \mathrm{M} P B S(\mathrm{pH}=7.4)$ on $\mathrm{GC} / \mathrm{CNT}$ electrode:

(a) SWVs at different DA concentrations; (b) calibration curve;

(c) $\mathrm{CVs}$ in $100 \mu \mathrm{M}$ DA solution at different scan rates;

(d) plot of $\log i_{p}$ versus $\log v$.

On GC/CNT electrode, the oxidation peak of AA is located at $-0.056 \mathrm{~V}$ (Figure 5a). The intensity of the oxidation peak increases with increasing concentration of $A A$ and its position shifts slightly from $-0.056 \mathrm{~V}$ to $-0.038 \mathrm{~V}$ with increasing concentration of $\mathrm{AA}$. The dependence between the intensity of the oxidation peak and AA concentration give a linear response (Figure 5b). 

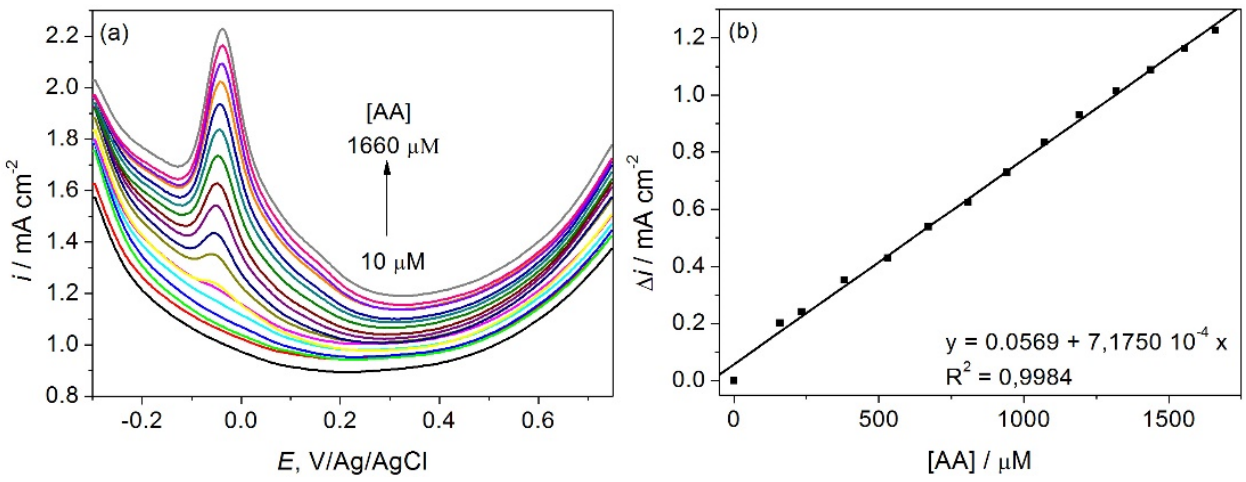

Figure 5. AA detection in 0.1 M PBS $(\mathrm{pH}=7.4)$ on $\mathrm{GC} / \mathrm{CNT}$ electrode:

(a) SWVs at different AA concentrations and (b) calibration curve.

The oxidation potential of AA on GC/CNT electrode is shifted by 450 $\mathrm{mV}$ to more negative values than on $\mathrm{GC}$, indicating its increased activity for AA oxidation. As a result, a sharp separation of the oxidation potentials of $A A$ and DA is obtained, which allows the simultaneous detection of the two molecules.
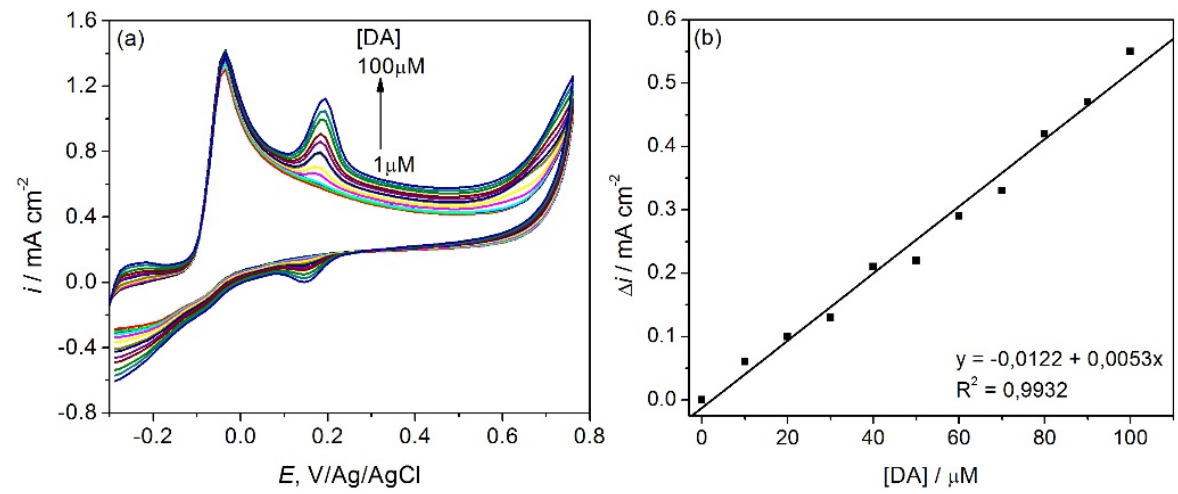

Figure 6. DA detection in the presence of $1.66 \mathrm{mM}$ AA in $0.1 \mathrm{M} \mathrm{PBS}(\mathrm{pH}=7.4)$ on GC/CNT: (a) cyclic voltammograms and (b) calibration curve.

This is evidenced in Figure 6, which shows cyclic voltammograms recorded for DA oxidation in the presence of AA at constant concentration $(1.66 \mathrm{mM})$. The first irreversible oxidation peak at $-0.038 \mathrm{~V}$ corresponds to AA oxidation and the second reversible peak at $0.190 \mathrm{~V}$ to DA oxidation, with a separation of $225 \mathrm{mV}$ between the two oxidation potentials. 
The detection range of DA in the presence of $A A(10$ to $100 \mu \mathrm{M})$ is lower as compared to DA detection in the absence of AA (2 to $100 \mu \mathrm{M})$ using the GC/CNT electrode, however the sensitivity remains similar and it is 20times higher than on GC electrode. The electroanalytical detection parameters are summarized in Table 2.

\section{Electrochemical detection of DA and AA on activated GC/CNT/ACT electrode}

A further improvement of the electrochemical detection parameters for DA detection was achieved by using activated CNT. By activation of the CNT (Figure 7a), carbonyl and / or carboxyl type functional groups are introduced on their surface, which act as reactive centers in the interaction with various molecules. The presence of these functional groups was sustained by FTIR spectra before and after activation (Figure $\mathbf{7 b}$ ).

(a)

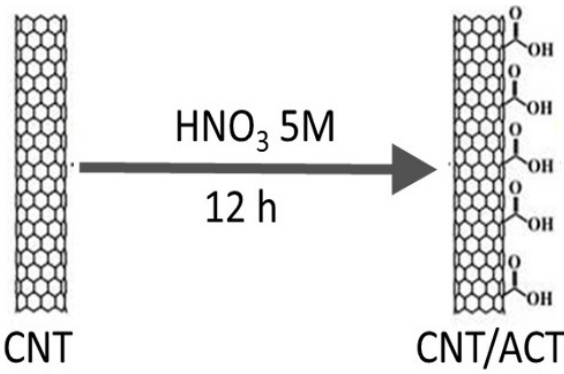

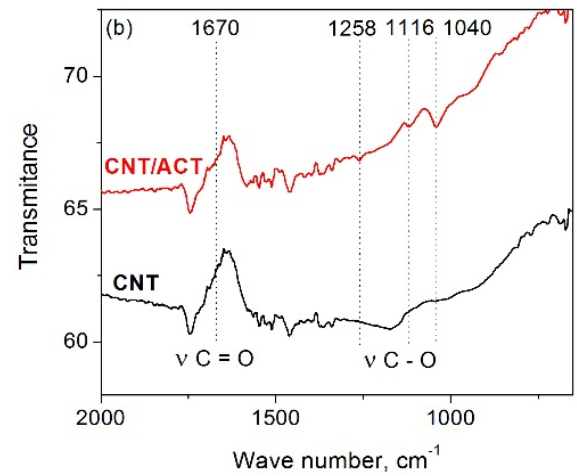

Figure 7. Activation of CNT: (a) Schematic representation and (b) FTIR spectra of pristine (CNT) and activated nanotubes (CNT/ACT).

The bands observed in the original CNT and CNT/ACT are assigned to the vibrations of carbon-oxygen groups from residual oxidation. The band at $1560 \mathrm{~cm}^{-1}$ is associated to $\mathrm{C}=\mathrm{C}$ stretching vibrations of the aromatic carbon in non-graphitic domains [19]. The broad band at $\sim 1130-1116 \mathrm{~cm}^{-1}$ has contributions from the skeletal $\mathrm{C}-\mathrm{C}$ observed in other carbon materials, and from some $\mathrm{C}-\mathrm{O}$ stretching vibrations of oxygen groups. A typical vibrational band of $\mathrm{OH}$ groups at $1340 \mathrm{~cm}^{-1}$, due to the $\mathrm{O}-\mathrm{H}$ bending mode. The shoulder at $1220 \mathrm{~cm}^{-1}$, is significantly more intense in the spectra of the oxidized carbon materials, when compared with the CNT. The FTIR spectra also showed vibrational bands related to the $\mathrm{C}=\mathrm{O}$ stretching of carboxylic acids, aldehydes, ketone and quinone groups at $1730 \mathrm{~cm}^{-1}$ and of ketones and quinones at 
$1670 \mathrm{~cm}^{-1}$. The CNT/ACT spectrum showed the presence of higher relative intensity bands associated with $v(\mathrm{C}=\mathrm{O})$ and $\delta(\mathrm{O}-\mathrm{H})$ vibrations of carboxylic acids (1730 and $1670 \mathrm{~cm}^{-1}$, respectively). These distinctions indicate a higher percentage of acid groups on the surface of these samples when compared to CNT. In addition, activation increases important parameters as the specific surface area and capacity, and open up new possibility of application of the carbon nanotubes (i.e. in energy storage devices).

The SWVs recorded on the activated GC/CNT/ACT electrode for different concentrations of AA (Figure 8a) shows that the intensity of oxidation peak of $A A$ increases and its position shifts slightly from $-0.05 \mathrm{~V}$ to $-0.022 \mathrm{~V}$ with increasing concentration of AA. Also, a linear dependence of peak intensity versus $A A$ concentration is obtained (Figure $\mathbf{8 b}$ ).
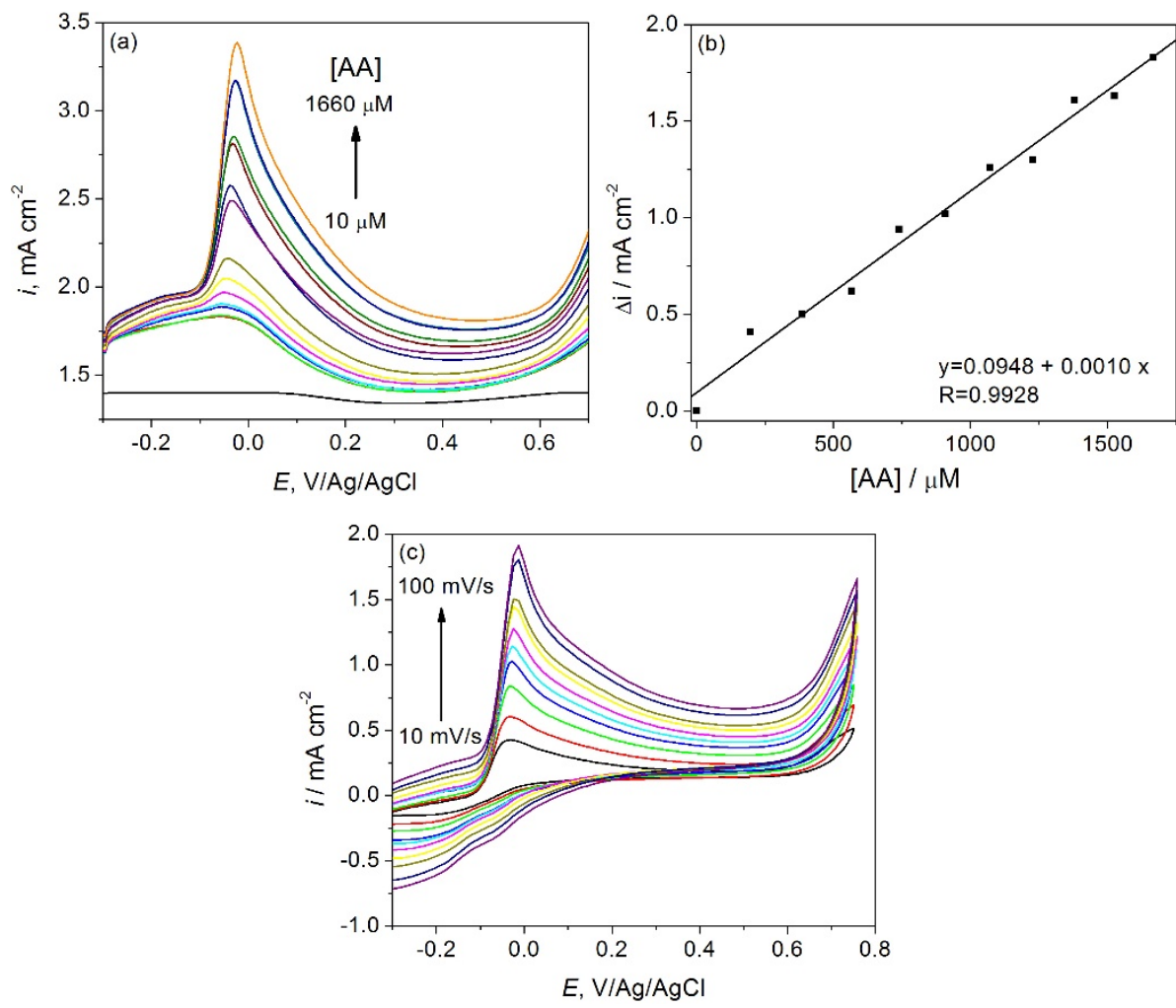

Figure 8. AA detection in $0.1 \mathrm{M} \mathrm{PBS}(\mathrm{pH}=7.4)$ on $\mathrm{GC} / \mathrm{CNT} / \mathrm{ACT}$ electrode:

(a) SWVs at different AA concentrations; (b) calibration curve and

(c) CVs in 2 mM AA solution at different scan rates. 
The AA oxidation reaction on GC/CNT/ACT electrode studied by cyclic voltammetry shows an irreversible oxidation peak that shifts from $-0.03 \mathrm{~V}$ to $-0.01 \mathrm{~V}$ as the scan rate increases (Figure 8c).

The SWVs recorded on the activated GC/CNT/ACT electrode for different DA concentrations are presented in Figure 9a.
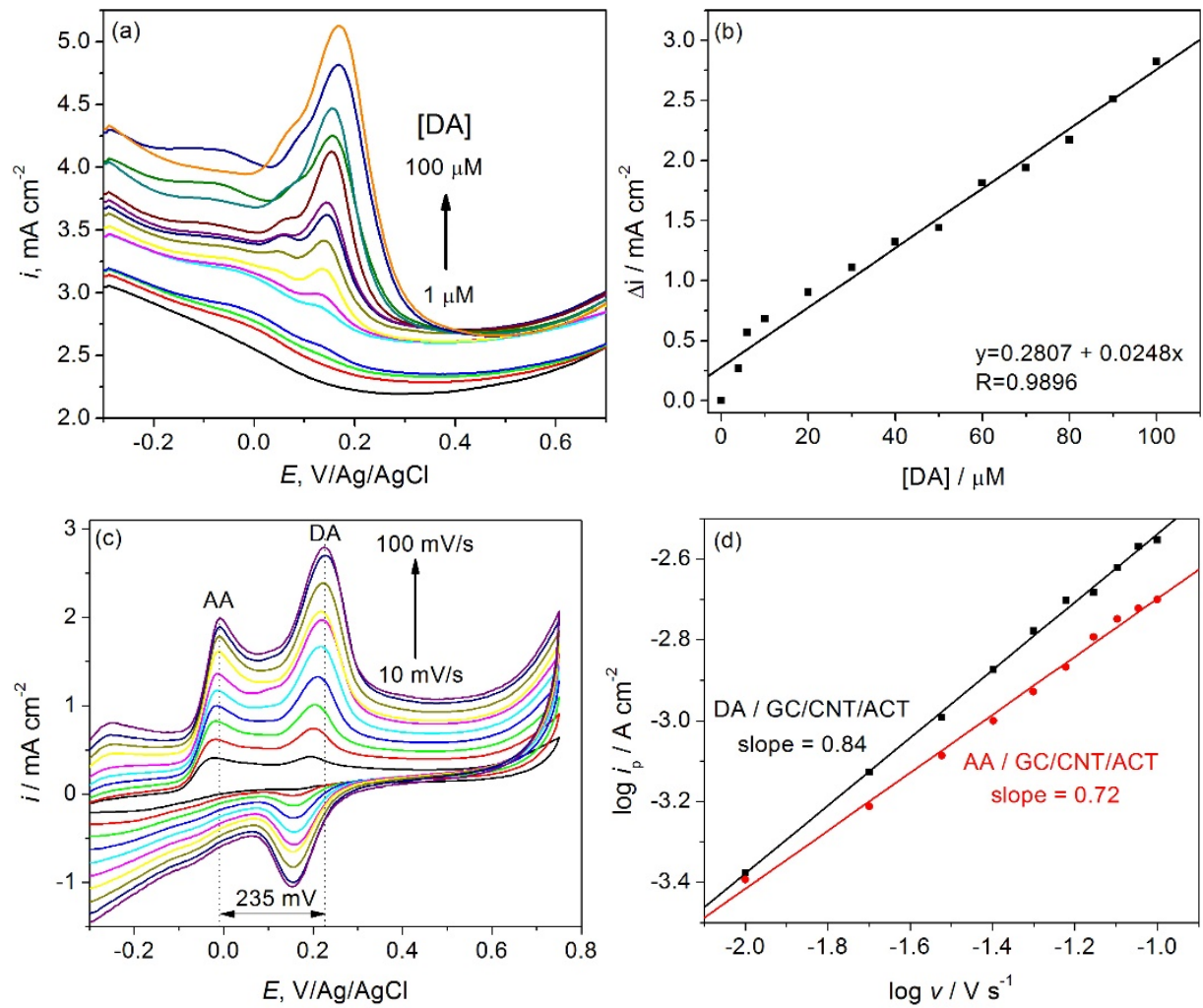

Figure 9. DA detection in $0.1 \mathrm{M} P B S(\mathrm{pH}=7.4)$ on $\mathrm{GC} / \mathrm{CNT} / \mathrm{ACT}$ electrode:

(a) SWVs at different DA concentrations; (b) calibration curve

(c) CVs of $0.1 \mathrm{mM}$ DA in the presence of $1.66 \mathrm{mM}$ AA at different scan rates;

(d) plot of $\log i_{p}$ versus $\log v$.

The intensity of the oxidation peak increases linearly (Figure 9b) and its position shifts slightly from $0.12 \mathrm{~V}$ to $0.16 \mathrm{~V}$ with increasing DA concentration. Cyclic voltammograms at different scan rates for a mixture of $1.66 \mathrm{mM}$ AA and $0.1 \mathrm{mM}$ DA are shown in Figure 9c. A peak separation of $235 \mathrm{mV}$ between the oxidation potentials of AA and DA is observed, which makes possible their simultaneous detection. A single peak of AA oxidation is observed, without a correspondent in the cathodic branch, which is specific 
to irreversible processes, while in the case of DA, two associated peaks are observed, indicating that DA oxidation is a reversible process. In addition, the peak current for DA oxidation shows a more pronounced increase with the scan rate compared to the peak current for AA oxidation, the peak current ratio being 1.4. The plot of $\log i_{\mathrm{p}}$ versus $\log v$ given in Figure $9 \mathrm{~d}$ also confirms this finding and shows different slopes for the oxidation of DA (0.840) and AA (0.716) on activated GC/CNT/ACT electrode, indicating an adsorptioncontrolled mechanism for DA oxidation and a mixed control of both diffusion and adsorption on AA oxidation. This behavior can be explained by enhanced attractive interactions between DA and the carbonyl and/or carboxyl type functional groups generated by CNT activation. DA presents an orthohydroxybenzene structure and an amino group in the side chain, protonated and positively charged at the working $\mathrm{pH}$ of 7.4 (pKa $=8.87$ [20]). Also, the presence of benzene ring in the DA structure makes the oxidation of DA more facile due to the adsorption through $\pi-\pi$ interactions of both its reduced and oxidized forms at $\mathrm{sp}^{2}$ carbons of the CNT/ACT modified electrode [21].

Table 1. Effect of scan rate on the voltammetric response of GC, GC/CNT and GC/CNT/ACT electrodes for the oxidation of DA and AA, separately or in a mixture.

\begin{tabular}{|l|l|l|l|}
\hline Electrode (Analyte) & Concentration & slope $^{*}$ & $\mathrm{R}^{2} / \mathrm{n}^{* *}$ \\
\hline GC (DA) & $100 \mu \mathrm{M}$ & 0.407 & $0.9835 / 9$ \\
\hline GC/CNT (DA) & $100 \mu \mathrm{M}$ & 0.930 & $0.9506 / 10$ \\
\hline GC/CNT/ACT (DA+AA) & $100 \mu \mathrm{M}$ DA + 1.66 mM AA & 0.840 & $0.9990 / 10$ \\
\hline GC (AA) & $1.66 \mathrm{mM}$ & 0.481 & $0.9958 / 9$ \\
\hline GC/CNT (AA) & $1.66 \mathrm{mM}$ & 0.520 & $0.9907 / 7$ \\
\hline GC/CNT/ACT (AA+DA) & $1.66 \mathrm{mM}$ AA + 100 $\mu \mathrm{M}$ DA & 0.716 & $0.9979 / 10$ \\
\hline
\end{tabular}

${ }^{*}$ slope of $\log i_{p}-\log v$ dependence for the anodic process

${ }^{* *} R^{2}$ is the coefficient of determination and $n$ the number of experimental points.

Square wave voltammetry measurements performed at constant $A A$ concentration $(1.660 \mathrm{mM})$, and at different DA concentration (2 to $100 \mu \mathrm{M})$ are shown in Figure 10a. It is observed that the separation between the oxidation potentials of AA and DA is maintained at $200 \mathrm{mV}$, which allows the detection of DA in the presence of $A A$. The linearity range of the calibration line for DA in the presence of AA (Figure 10b) is maintained within the same limits as in the case of the calibration line for DA in the absence of $A A$, being between 6 and $100 \mu \mathrm{M}$. There is a slight decrease in sensitivity from $24.6 \mu \mathrm{A}$ / $\mu \mathrm{M} \mathrm{cm}{ }^{2}$ in the absence of AA to $19.2 \mu \mathrm{A} / \mu \mathrm{M} \mathrm{cm}{ }^{2}$ in the presence of $A A$, however DA can be detected selectively and sensitively using the activated $\mathrm{GC} / \mathrm{CNT} / \mathrm{ACT}$ electrode, even in the presence of concentrations of AA over 200 times higher. 

DOPAMINE IN THE PRESENCE OF ASCORBIC AND URIC ACID
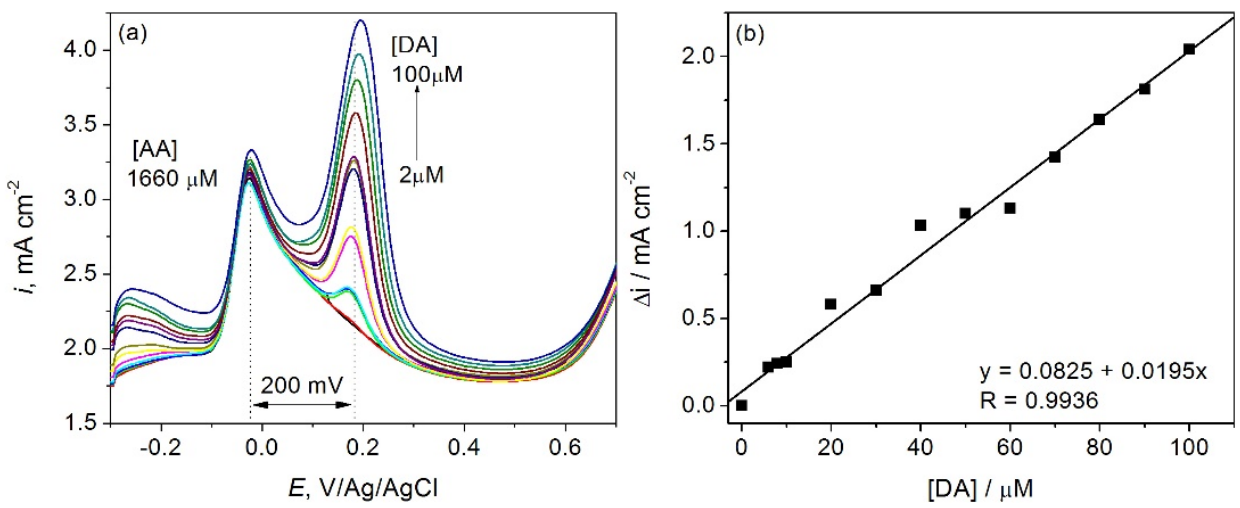

Figure 10. DA detection in the presence of $1.66 \mathrm{mM}$ AA in $0.1 \mathrm{M} \mathrm{PBS}(\mathrm{pH}=7.4)$ on activated GC/CNT/ACT electrode: (a) SWVs at different DA concentrations;

(b) Calibration line for DA detection in the presence of AA.

The result obtained for DA detection on GC/CNT and activated GC/CNT/ACT reveal comparable results with those reported for Polydopamine (PD) Copper(II) ions glassy carbon electrode (PD-Cu(II)h /GCE h Polydopamine) [20] or reduced graphene oxide and graphene oxide modified glassy carbon electrodes protected with a polymeric layer of Nafion or chitosan [24]. The detection parameters are better than those reported for Carbon fiber electrode (CFE) modified by graphene flowers (GEFi /CFE) [22]. Electrochemically pretreated pencil graphite electrode PGE [23] and nano-Au self-assembled modified electrode (nano-Au/CA/GC) [24] present lower detection limits than GC/CNT and GC/CNT/ACT.

The limit of detection (LOD) and limit of quantification (LOQ) were determined based on the standard deviation (S) and the slope of the calibration line $(\mathrm{m})$, according to the following equations: $L O D=3 \mathrm{~S} / \mathrm{m}$ and $L O Q=10 \mathrm{~S} / \mathrm{m}$. Table 2 summarizes the electroanalytical detection parameters of DA, including relative standard deviation (RSD), determined in the absence and presence of AA for all the investigated electrodes.

Table 2. Electroanalytical detection parameters for DA on GC, GC/CNT and activated GC/CNT/ACT electrodes.

\begin{tabular}{|l|r|r|c|r|r|r|}
\hline \multicolumn{1}{|c|}{ Electrode } & $\begin{array}{c}\text { Range, } \\
\mu \mathrm{M}\end{array}$ & $\begin{array}{c}\text { Sensitivity } \\
\mu \mathrm{A} / \mu \mathrm{M} \mathrm{cm} \mathrm{cm}^{2}\end{array}$ & $\mathrm{R}^{2}$ & $\begin{array}{c}\mathrm{RSD} \\
\%\end{array}$ & $\begin{array}{c}\mathrm{LOD} \\
\mu \mathrm{M}\end{array}$ & $\begin{array}{c}\mathrm{LOQ} \\
\mu \mathrm{M}\end{array}$ \\
\hline $\mathrm{GC}(\mathrm{DA})$ & $1 \ldots 100$ & 0.280 & 0.9997 & 0.019 & 16.08 & 53.59 \\
\hline GC/CNT(DA) & $2 \ldots 100$ & 3.730 & 0.9995 & 0.027 & 1.76 & 5.87 \\
\hline GC/CNT (DA+AA) & $10 \ldots 100$ & 5.300 & 0.9932 & 0.268 & 3.18 & 10.59 \\
\hline GC/CNT/ACT (DA) & $4 \ldots 100$ & 24.79 & 0.9896 & 0.021 & 0.44 & 1.45 \\
\hline GC/CNT/ACT (DA+AA) & $6 \ldots 100$ & 19.50 & 0.9936 & 0.023 & 0.64 & 2.14 \\
\hline
\end{tabular}




\section{Electrochemical detection of DA on CG/CNT/ACT electrode from synthetic biological samples in the presence of interferences}

The performance of GC/CNT/ACT electrodes for DA detection from biological samples in the presence of potential interferences, was verified by recording SWVs for DA in the presence of AA and UA in $0.1 \mathrm{M}$ PBS solution and by performing recovery measurement in a Ringer solution in the presence of AA and UA. Figure 11 shows SWVs on activated GC/CNT/ACT for a solution containing $2 \mathrm{mM} \mathrm{AA}, 0.1 \mathrm{mM}$ DA and $0.5 \mathrm{mM} \cup \mathrm{A}$.

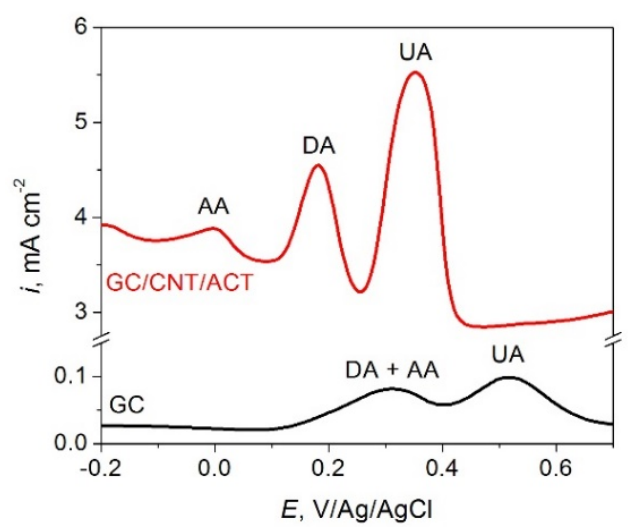

Figure 11. SWVs for a mixture of $2 \mathrm{mM} \mathrm{AA}, 0.1 \mathrm{mM} D A$ and $0.5 \mathrm{mM}$ UA on GC and activated GC/CNT/ACT electrode.

The electrochemical response of GC/CNT/ACT electrode is characterized by the appearance of three distinct oxidation peaks, corresponding to the oxidation of AA, DA and UA. The same measurements performed on the unmodified GC electrode indicate the appearance of only two oxidation peaks, the first one attributed to the simultaneous oxidation of AA and DA, and the second to the oxidation of UA. The peak potentials for the oxidation of DA, AA and UA on GC and GC/CNT/ACT are listed in Table 3. On the $\mathrm{GC} / \mathrm{CNT} / \mathrm{ACT}$ electrode, it is possible to detect DA in the presence of AA and $U A$, the differences between the peak potentials being of $0.20 \mathrm{mV}$ and $0.17 \mathrm{mV}$, respectively.

Table 3. Peak potentials for simultaneous oxidation of DA, AA and UA on GC and activated GC/CNT/ACT electrodes in $0.1 \mathrm{M}$ PBS.

\begin{tabular}{|l|c|c|c|}
\hline Electrode & $E_{\mathrm{AA}}[\mathrm{V}]$ & $E_{\mathrm{DA}}[\mathrm{V}]$ & $E_{\mathrm{UA}}[\mathrm{V}]$ \\
\hline $\mathrm{GC}$ & 0.32 & 0.32 & 0.52 \\
\hline GC/CNT/ACT & -0.02 & 0.18 & 0.35 \\
\hline
\end{tabular}


For the recovery measurements, a Ringer's solution was used, with added $\mathrm{AA}$ and UA in order to obtain a concentration of $20 \mu \mathrm{M}$ of each electroactive species. Then SWVs were recorded, with successive additions of DA to have increasing concentrations of $40,50,60,70$ and $80 \mu \mathrm{M}$. The obtained results are given in Table 4 and indicate satisfactory levels of recovery in the investigated concentration range.

Table 4. DA detection in Ringer's solution in the presence of AA and UA.

\begin{tabular}{|c|c|c|}
\hline Added $[\mu \mathrm{M}]$ & Detected $[\mu \mathrm{M}]$ & Recovery $[\%]$ \\
\hline 40 & 31.8 & 79.5 \\
\hline 50 & 49.3 & 98.5 \\
\hline 60 & 59.0 & 98.4 \\
\hline 70 & 65.9 & 94.2 \\
\hline 80 & 75.3 & 94.1 \\
\hline
\end{tabular}

\section{CONCLUSIONS}

The kinetics of DA and AA oxidation reaction was studied by cyclic voltammetry on GC, GC/CNT and activated GC/CNT/ACT electrodes. It has been found that DA oxidation on $\mathrm{GC}$ is a diffusion-controlled process, but it changes to an adsorption-controlled process on GC/CNT and GC/CNT/ACT electrodes.

The separate detection of DA and AA was carried out by square wave voltammetry, at concentrations between $1 \ldots 100 \mu \mathrm{M}$ for DA and $10 \ldots 1660 \mu \mathrm{M}$ for $A A$ and the electroanalytical detection parameters were determined. The sensitivity of DA detection increased from $0.28(\mathrm{GC})$ to 3,73 (GC/CNT) and $24.79 \mu \mathrm{A} / \mu \mathrm{M} \mathrm{cm}^{2}$ (GC/CNT/ACT). On GC electrode, the oxidation potentials of $D A$ and $A A$ are close, therefore DA detection in the presence of $A A$ is not feasible. However, it has been found that modification of GC electrode with CNT and CNT/ACT induces a potential peak separation of 235 and $200 \mathrm{mV}$, respectively, which allows detection of $D A$ even in the presence of $A A$, at more than 100 times higher concentrations. In the presence of $1.66 \mathrm{mM} \mathrm{AA}$, the sensitivity slightly decreases to $19.50 \mu \mathrm{A} / \mu \mathrm{M} \mathrm{cm}{ }^{2}$ on GC/CNT/ACT, compared to $24.79 \mu \mathrm{A} / \mu \mathrm{M} \mathrm{cm}{ }^{2}$ in the absence of AA. In conclusion DA can be selectively and sensitively detected using GC modified with activated CNT.

GC/CNT/ACT electrodes also showed very good performance in the presence of both $A A$ and $U A$, with well-resolved potential peak separation $\left(\Delta E_{\mathrm{AA}-\mathrm{DA}}=200 \mathrm{mV}, \Delta E_{\mathrm{DA}-\mathrm{UA}}=170 \mathrm{mV}\right.$ and $\left.\Delta E_{\mathrm{AA}-\mathrm{UA}}=370 \mathrm{mV}\right)$.

Measurements of DA detection in synthetic solutions (Ringer's solution) in the presence of $A A$ and UA showed that the method is reliable, obtaining recovery rates of $98 \%$ for added concentrations of DA of 50 and $60 \mu \mathrm{M}$, 
respectively. In conclusion, our results showed the importance of activation step in the construction of CNT-based sensors for DA. The activated GC/CNT/ACT electrode can be successfully used for DA detection in the range of micromolar concentrations.

\section{EXPERIMENTAL SECTION}

\section{Materials}

Phosphate buffer (PBS) was prepared and used as the electrolyte solution, with the following composition: $\mathrm{NaCl} 0.8 \mathrm{~g} \mathrm{~L}^{-1}, \mathrm{KCl} 0.2 \mathrm{~g} \mathrm{~L}^{-1}$, $\mathrm{Na}_{2} \mathrm{HPO}_{4} 1.42 \mathrm{~g} \mathrm{~L}^{-1}, \mathrm{KH}_{2} \mathrm{PO}_{4} 0.24 \mathrm{~g} \mathrm{~L}^{-1}$ and distilled water to $1 \mathrm{~L}$. All chemicals $\mathrm{NaCl}, \mathrm{KCl}, \mathrm{Na}_{2} \mathrm{HPO}_{4}, \mathrm{KH}_{2} \mathrm{PO}_{4}$ were purchased from Merck and used without purification. Stock solutions of $10^{-2} \mathrm{M}$ dopamine ( $\geq 98.5 \%$ Fluka), $10^{-2} \mathrm{M} \mathrm{L-}$ ascorbic acid ( $\geq 99 \%$, Sigma Aldrich) and $10^{-2}$ uric acid $(\geq 99 \%$, Sigma Aldrich) were freshly prepared in PBS buffer before use. A Ringer solution from $\mathrm{B}$. Braun, with the composition $\mathrm{NaCl} 8.60 \mathrm{~g} \mathrm{~L}^{-1}, \mathrm{KCl} 0.30 \mathrm{~g} \mathrm{~L}^{-1}$ and $\mathrm{CaCl}_{2}$ $2 \mathrm{H}_{2} \mathrm{O} 0.33 \mathrm{~g} \mathrm{~L}^{-1}$ was used. Multi-walled carbon nanotubes from Sigma Aldrich with an outer diameter of 6-9 $\mathrm{nm}$ and a length of $5 \mu \mathrm{m}$ were used to modify the working electrode. As working electrode a glassy carbon electrode (GC) was used. Prior to each use, the electrode surface was prepared by polishing using diamond suspension with a particle size of 6 and $3 \mu \mathrm{m}$, respectively, followed by thoroughly rinsing with distilled water.

\section{CNT activation and characterization}

To activate the carbon nanotubes, $0.5 \mathrm{~g}$ of CNT were weighted and kept for 12 hours in $5 \mathrm{M} \mathrm{HNO}_{3}$ under magnetic stirring. The resulting product was then filtered and washed with abundant distilled water to neutral $\mathrm{pH}$, followed by drying in an oven at $70^{\circ} \mathrm{C}$ for 12 hours. Samples are denoted as CNT/ACT. The structure of pristine and activated CNT was investigated by Fourier-transform infrared spectroscopy (FTIR), using an IR Prestige 21 Shimadzu spectrophotometer. FTIR spectra were recorded in $\mathrm{KBr}$ pellets, for wave numbers between 400 and $4000 \mathrm{~cm}^{-1}$, where each spectrum consists of 20 interferograms with a resolution of $4 \mathrm{~cm}^{-1}$.

\section{GC/CNT and GC/CNT/ACT electrode preparation}

A suspension of $4 \mathrm{mg}$ CNT or CNT/ACT in $2 \mathrm{~mL}$ ethanol was subjected to ultrasonication for 60 minutes. The modified electrodes were prepared by drop casting $10 \mu \mathrm{L}$ of the CNT or CNT/ACT suspension onto the previously cleaned surface of the GC, followed by solvent evaporation under nitrogen flow. 


\section{Electrochemical measurements}

Cyclic voltammetry and square wave voltammetry measurements were performed in a three-electrode configuration electrochemical cell, using an AUTOLAB PGSTAT 302N. The electrochemical cell $(50 \mathrm{~mL})$ was equipped with a working glassy carbon electrode (GC, $\Phi=3 \mathrm{~mm}$, Metrohm A.G., Switzerland), a Pt wire electrode as a counter electrode and the reference electrode was a silver-silver chloride electrode, $\mathrm{Ag} / \mathrm{AgCl}$. Electrodes were immersed in $25 \mathrm{~mL}$ of PBS electrolyte solution and a SWV was recorded for the supporting electrolyte in the absence of DA and / or AA. SWV was carried with pulse amplitude of $20 \mathrm{mV}$, frequency of $10 \mathrm{~Hz}$ and step potential of $5 \mathrm{mV}$. $\mathrm{CVs}$ were recorded at increasing scan rates, from 10 to $100 \mathrm{mV} \mathrm{s}^{-1}$, in steps of $10 \mathrm{mV} \mathrm{s}^{-1}$. For the detection of DA and AA, SWV measurements were performed in the concentration range of $1-100 \mu \mathrm{M}(\mathrm{DA})$ and $10-1660 \mu \mathrm{M}$ (AA), respectively. Successive additions from the stock solutions were made of $2.5,5,10,15,25,50,75,100,125,150,175,200,225$ and $250 \mu \mathrm{L}$ for DA and $25,50,100,200,400,600,1000,1400,1800,2200,2600,3000,3400$, $3800,4200,4600$ and $5000 \mu \mathrm{L}$ for AA. The corresponding concentration were calculated considering the volume change of the final solution. The calibration lines were obtained by representing $\Delta i$ versus concentration of DA or AA, were $\Delta i$ represents the difference between the current in the presence and absence of the analyte, at the same potential value.

\section{REFERENCES}

1. L. Yang, D. Liu, J. Huang, T. You, Sensor. Actuat. B-Chem, 2014, 193, 166-172

2. N.D. Oko, S. Garbarino, J. Zhang, Z. Xu, M. Chaker, D. Ma, D. Guay A.C. Tavares, Electrochim. Acta, 2015, 159, 174-183.

3. R.T. Kennedy, C.J. Watson, W.E. Haskins, D.H. Powell, R.E Strecker, Curr. Opin. Chem. Biol., 2002, 6, 659-665.

4. B.J. Venton, R.M. Wightman, Anal. Chem., 2003, 75, 414A-421A.

5. N.G. Tsierkezosa, S.H. Othman, U. Ritter, L. Hafermann, A. Knauer, J.M. Köhler, C. Downing, E.K. McCarthy, Sensors Actuat. B-Chem., 2016, 231, 218-229.

6. N. Chauhan, S. Chawla, C.S. Pundir, U. Jain, Biosens. Bioelectron., 2017, 89, 377-383.

7. A. Ramanavicius, A. Ramanaviciene, A. Malinauskas, Electrochim. Acta 2006, $51,6025-6037$.

8. D. Wu, H. Li, X. Xue, H. Fan, Q. Xin, Q. Wei, Anal. Methods., 2013, 5, 1469-1473. 
9. N. Plesu, A. Kellenberger, I. Taranu, B.O Taranu, I. Popa, I., React. Funct. Polym., 2013, 73 (5), 772-778.

10. X. Zhang, L.-X. Ma, Y.-C. Zhang, Electrochim. Acta, 2015, 177, 118-127.

11. A.K. Bhakta, R.J. Mascarenhas, O.J. D'Souza, A.K. Satpati, S. Detriche, Z. Mekhalif, J. Dalhalle, Mater. Sci. Eng. C., 2015, 57, 328-337.

12. E.C. llinoiu, F. Manea, P.A. Serra, R. Pode, Sensors, 2013, 13(6), 7296-7307.

13. C. Wang, J. Du, H. Wang, C. Zou, F. Jiang, P.Yang, Y. Du, Sensor. Actuat. B -Chem., 2014, 204, 302-309.

14. F.R. Caetano, L.B. Felippe, A.J.G. Zarbin, M.F. Bergamini, L.H. MarcolinoJunior, Sensor. Actuat. B-Chem., 2017, 243, 43-50.

15. I.V. Zaporotskova, N.P. Boroznina, Y.N. Parkhomenko, L.V. Kozhitov, Mod. Electron. Mater., 2016, 2, 95-105.

16. M.J. Allen, V.C. Tung, R.B. Kaner, Chem. Rev., 2010, 110, 132-145

17. Q. He, J. Liu, X. Liu, G. Li, P. Deng, J. Liang, Sensors, 2018, 18, 199;

18. Z. Hsine, S. Bizid, R. Mlika, H. Sauriat-Dorizon, A. Haj Said, H. Korri-Youssoufi, Sensors 2020, 20, 1256;

19. H. Gaspar, C. Pereira, S.L.H. Rebelo, M.F.R. Pereira, J.L. Figueiredo, C. Freire, Carbon, 2011, 49, 3441-3453.

20. J. Armstrong, R.B. Barlow, Br. J. Pharmacol., 1976, 57, $501-516$

21. R. Gusmão, M. Melle-Franco, D. Geraldo, F. Bento, M.C. Paiva, F. Proença, Electrochem. Commun., 2015, 57, 22-26.

22. A. Liu, M.D. Wei, I. Honma, H. Zhou, Adv. Funct. Mater., 2006, 16, 371 - 376.

23. L. Huang, S. Jiao, M. Li, Electrochim. Acta, 2014, 121, 233-239.

24. A.F. Szőke, G.L. Turdean, G. Katona, L. M. Muresan, Studia UBB Chemia, 2016, 3, 135-144.

25. J. Du, R. Yue, F. Ren, Z. Yao, F. Jiang, P. Yang, Y. Du, Biosens. Bioelectron., 2014, 53, 220-224.

26. D.C. Stefanescu A.A. Ciucu, A.A. Rabinca, M. Buleandra, A.P. Stoian, R.C. Jecan, R. Hainarosie, Rev.Chim., 2018, 69(1), 277-281.

27. G.-Z. Hu, D.-P. Zhang, W.-L. Wu, Z.S. Yang, Colloids Surf. B: Biointerfaces, 2008, 62, 199-205. 